\title{
ON INTEGRAL EQUATIONS OF THE FIRST KIND WITH LOGARITHMIC KERNELS
}

\author{
Y. YAN AND I.H. SLOAN
}

\begin{abstract}
The existence-uniqueness of the solution and its behaviour for one-dimensional integral equations of the first kind with logarithmic kernels are investigated. The analysis is based on the transfinite diameter or logarithmic capacity and spaces deriving from Fourier series. The uniqueness results apply to any closed bounded subset of the plane. The other results apply to open arcs, polygons and other regions with piecewise-smooth boundaries.
\end{abstract}

0. Introduction. In this paper, the integral equation of the first kind,

$$
-\int_{\Gamma} \log |x-y| g(y) d y=f(x), \quad x \in \Gamma,
$$

will be investigated, where $d y$ denotes the arc-length element at $y \in \Gamma$. $\Gamma$ is a curve in the plane, smooth or piecewise smooth, open or closed, such as an interval, circle or polygon [5]. Some discussion will also be given for the more general case, appearing in $[\mathbf{2 4}],[\mathbf{2}],[\mathbf{3 8}]$,

$$
-\int_{\Gamma}[\log |x-y|+F(x, y)] g(y) d y=f(x), \quad x \in \Gamma,
$$

where $F(x, y)$ is a function of $x, y \in \Gamma$ which is smoother than $\log |x-y|$. We will give existence-uniqueness theorems for the solutions and show their smoothness and singular character.

The integral equation of the first kind in the form (1) follows from the representation of a harmonic function by single-layer potentials, or by the direct boundary integral equation method (BIEM) for plane Dirichlet boundary value problems, both of great importance in engineering $[29,4, \mathbf{1 8}, 5,6,7]$. The classical boundary integral equations usually appeared in the form of second kind integral equations [34, 29], because of the simplicity of Fredholm theory. But in the last decade engineers and mathematicians have noticed that boundary integral equations of 
the first kind with logarithmically singular kernels allow simple numerical solutions of practical problems. By now much attention has been paid to their numerical analysis.

On the theoretical side, this kind of equation cannot fit within the framework of the classical Fredholm theory and is in some ways more difficult than the traditional integral equation of the second kind $[\mathbf{3}]$. The reason is that the logarithmic operator has a smoothing effect and typically maps a function space to a smaller space. As a result, an analysis of equation (1) in a single function space will usually result in solutions failing to exist for some function $f(x)$, and hence instability.

Another integral equation

$$
-\int_{\Gamma} \log |x-y| g(y) d y+\omega=f(x), \quad x \in \Gamma, \quad \int_{\Gamma} g(y) d y=0
$$

has a close relation to (1) [41]. This equation has been used frequently in single-layer potential theory $[\mathbf{2 4}, \mathbf{2 6}, \mathbf{2}]$. When $\Gamma$ is a smooth boundary of a bounded simply connected domain $\Omega$ both (1) and (2) have been included in the abstract theoretical framework of pseudodifferential operators $[39,44,45]$. This has turned out to be a useful tool for deriving error estimates for the Galerkin and collocation methods for boundary integral equations $[25,27,44,45,46,28,2$, 38]. When $\Gamma$ is a polygonal boundary, the pseudo-differential operator theory is not directly applicable. The integral operator no longer has a convolutional principal part and the solution of the integral equation in general has singularities at the corners $[30,20,29,12,21,41,47]$ even if the right hand side of the equation is smooth. For this case, Mellin transforms have been used $[\mathbf{1 0}, \mathbf{1 1}, \mathbf{1 2}, \mathbf{1 3}, \mathbf{1 4}, \mathbf{1 5}]$ to analyse the boundary integral equation and have yielded error estimates for the Galerkin and collocation methods. In either case, a central idea of these works is the proof of strong ellipticity of the integral operator. One technique used in that proof is the trace theorem. This technique appeared first in the work of Hsiao and Wendland [25], where the existence and uniqueness of solutions for (1) and (2) were proved under the condition of a smooth boundary and with the problem scaled so that diameter $(\Omega)<1$. Also, later in the works of Costabel and Stephan $[12,15,16]$, the existence and uniqueness of (1) were proved for a polygonal or Lipschitz boundary. Another technique is Fourier analysis, which has been used to prove the existence and uniqueness of solutions 
for (2) with $\Gamma$ a smooth boundary $[\mathbf{2 7}, \mathbf{9}, \mathbf{3 3}]$. The existence and uniqueness of solutions for (1) and (2) have also been analysed without showing the strong ellipticity by Verchota $[\mathbf{4 3}]$ and McLean $[\mathbf{3 3}]$ in whose work $\Gamma$ is a closed Lipschitz contour. For the case of a $\Gamma$ with less restriction (e.g., $\Gamma$ an open arc), the energy integral method has been used to generate error estimates for the Galerkin method $[\mathbf{4 1}, \mathbf{4 7}]$.

Whereas the trace theorem is restricted to cases in which $\Gamma$ is the boundary of a bounded simply connected domain, Fourier analysis may be available for both open arcs and closed contours. This is the path we follow in this paper. We shall assume $\Gamma$ to be a simple curve (open or closed) except in the general discussion in $\S 1$.

The behaviour of the solution of (1) depends on whether $\Gamma$ is closed or open. Loosely speaking, when $\Gamma$ is smooth and closed, the smoother $f(x)$ is, the smoother the solution $g(x)$ is. If $\Gamma$ is open, then usually $g(x)$ is singular at the two end points of $\Gamma$, no matter how smooth $f(x)$ is. Hence the structure of the solution on the different contours, closed or open, is quite different. For this reason we shall analyse the cases of closed and open contours separately, writing the contour as $\Gamma_{c}$ in the closed case, and $\Gamma_{o}$ in the open.

In the case of the open (smooth) contour, we shall introduce a simple cosine change of variable (see $\S 4$ ), which renders the analysis similar to that for the closed contour and makes the singularities at the ends explicit. This novel approach to the open arc is one of this paper's main contributions.

To enhance understanding, we adopt a somewhat expository style. Thus in much of this paper we consider in parallel both closed curves, for which many of the results are known, and open arcs, for which the results are new.

In $\S 1$ we collect some useful results from classical potential theory and prove a uniqueness theorem which plays a key role in the analysis. In $\S 2$ and 3 we develop Fourier series representations of two special integral operators with logarithmic kernels. One is appropriate to smooth closed curves, the other (after a change of variable) to open arcs. The setting for the first is a standard Sobolev space $H^{t}$, for the second a slightly modified space $H_{e}^{t}$.

The main results are presented in $\S 4$ and 5 . In $\S 4$ a theoretical analysis is given for both closed and open contours $\Gamma$ and existence 
and uniqueness theorems are proved. In $\S 5$ existence and uniqueness are analysed for the case of a polygonal contour $\Gamma$ by means of a perturbation argument applied to the results for smooth closed curves.

1. Results from potential theory. In this section the results apply to a very general class of contour $\Gamma$. Assume only that $\Gamma$ is a closed, bounded set in $\mathbf{R}^{2}$. Correspondingly, the language is that of measures and charges on $\Gamma$. However, if $\Gamma$ is suitably regular a correspondence with equation (1) may be obtained by replacing $d \mu$ or $d \sigma$ below by $g(y) d y$.

The methods used are those of classical potential theory, rather than those of partial differential equations. As in $[\mathbf{4 1}]$, an essential concept is that of the "transfinite diameter", or the equivalent concept of the "logarithmic capacity" $C_{\Gamma}$, as discussed by Hille [23].

The most important result of this section is Theorem 1.3 which establishes that the solution of (1) is unique, even if the equation is interpreted in the most general measure-theoretic sense. The only restrictions are that $C_{\Gamma} \frac{1}{\tau} 1$ (or, in the language of $[\mathbf{2 9}]$, that the contour is not a $\Gamma$-contour) and that the solution has "finite energy".

Let $\Gamma$ be a bounded closed subset of the plane and let $M(\Gamma)$ denote the set of normalized measures $\mu$, i.e., $\mu$ is a measure such that $\mu(\Gamma)=1$ and $0 \leq \mu(S) \leq 1$ for $S \subset \Gamma$.

For $\mu \in M(\Gamma)$, the "energy integral" $I(\mu)$ is defined by

$$
I(\mu)=\int_{\Gamma} \int_{\Gamma} \log |x-y|^{-1} d \mu(x) d \mu(y) .
$$

The energy integral always exists, but may have the value $+\infty$.

We now define $V_{\Gamma}$ (the "Robin constant" of the set $\Gamma$ ) by

$$
V_{\Gamma}=\inf _{\mu \in M(\Gamma)} I(\mu),
$$

which satisfies $V_{\Gamma}>-\infty$ (see $[\mathbf{2 3}]$ ). The logarithmic capacity $C_{\Gamma}$ is then defined by

$$
C_{\Gamma}=e^{-V_{\Gamma}} .
$$

(The transfinite diameter of $\Gamma$ is defined differently, see $[\mathbf{2 3}]$, but Hille shows that the two concepts are equivalent. We shall use the phrase "transfinite diameter".) 
Some useful properties of the transfinite diameter are:

1. $C_{\Gamma} \leq d_{\Gamma}$, where $d_{\Gamma}$ is the Euclidean diameter of $\Gamma$.

2. If $\Gamma=\alpha \Gamma_{1}$, then $C_{\Gamma}=\alpha C_{\Gamma_{1}}$.

3 . If $\Gamma$ is the "outer boundary" $[\mathbf{2 3}]$ of a closed bounded set $E$, then $C_{\Gamma}=C_{E}$.

4. If $E_{1} \subset E_{2}$, then $C_{E_{1}} \leq C_{E_{2}}$.

5. The transfinite diameter of an interval of length $a$ is (1/4)a.

6. The transfinite diameter of a circle of radius $a$ is $a$.

The following result (see $[\mathbf{2 3}, \mathbf{3 1}]$ ) often allows subsets of "capacity zero" to be ignored in an integration.

LEMMA 1.1. Let $\Gamma$, a closed bounded set with $C_{\Gamma}>0$, have a subset $S \subset \Gamma$ such that $C_{S}=0$. If $\mu \in M(\Gamma)$ and $I(\mu)<\infty$, then $\mu(S)=0$.

Here $C_{S}=0$ indicates, as in $[\mathbf{3 1}]$, that for any compact set $E \subset$ $S, C_{E}=0$.

Another important result proved by Hille follows.

LEMMA 1.2. If $C_{\Gamma}>0$, then there exists a unique measure $\mu_{e} \in M(\Gamma)$ that achieves the infimum in (4).

The significance of the "equilibrium distribution" $\mu_{e}$, which satisfies $V_{\Gamma}=I\left(\mu_{e}\right)$, will be apparent from the next result. Define

$$
U(x, \mu)=\int_{\Gamma} \log |x-y|^{-1} d \mu(y), \quad x \in \mathbf{R}^{2},
$$

the "potential" at $x$ arising from the measure $\mu$ on $\Gamma$. It is known that $U(x, \mu)$ is a harmonic function for $x$ in the complement of $\operatorname{supp}(\mu)$ (excluding the point at infinity). The following result $[\mathbf{3 1}]$ concerns the potential arising from the equilibrium distribution $\mu_{e}$.

LEMMA 1.3. Let $\Gamma$ be a closed bounded set of positive transfinite diameter $C_{\Gamma}$. Then $U\left(x, \mu_{e}\right) \leq V_{\Gamma}$ in the whole plane, and for $x \in \Gamma$ we have $U\left(x, \mu_{e}\right)=V_{\Gamma}$ except possibly in a subset of capacity zero. 
Thus the equilibrium distribution gives rise (except possibly on a subset of capacity zero) to a constant potential on $\Gamma$.

The definitions of the energy integral (3) and the potential (5) may be extended from measures to signed "charges" (i.e., completely additive set functions). Every charge $\sigma$ can be written (see [17]) as $\sigma=\sigma_{+}-\sigma_{-}$, where $\sigma_{ \pm}$are measures. Let $\mathcal{S}(\Gamma)$ be the set of charges $\sigma$ for which $I(|\sigma|)<\infty$, where $|\sigma|=\sigma_{+}+\sigma_{-}$. (Thus $\mathcal{S}(\Gamma)$ is the set of charges with "finite energy".) Then $I(\sigma)$ and $U(x, \sigma)$, obtained from (3) and (5) by replacing $\mu$ by $\sigma$, are well defined for $\sigma \in \mathcal{S}(\Gamma)$.

Lemma 1.1 extends in an obvious way to charges.

LEMMA 1.4. Let $\Gamma$, a closed bounded set with $C_{\Gamma}>0$, have a subset $S$ such that $C_{S}=0$. If $\sigma \in \mathcal{S}(\Gamma)$, then $|\sigma|(S)=0$.

Charges $\sigma$ with finite energy and with the property that the total charge $\sigma(\Gamma)$ is zero lead to particularly simple results. For the following important result, see $[\mathbf{1 7}]$.

Lemma 1.5. Let $\sigma \in \mathcal{S}(\Gamma)$ be such that $\sigma(\Gamma)=0$. Then $I(\sigma) \geq 0$, with equality if and only if $\sigma=0$.

From this result follows, as in $[\mathbf{4 1}]$, the known positive-definite property of the bilinear form $I(\sigma)$, provided $C_{\Gamma}<1$.

THEOREM 1.1. (positive definiteness). Let $\Gamma$ be a closed bounded set, with transfinite diameter satisfying $0<C_{\Gamma}<1$. Assume $\sigma \in \mathcal{S}(\Gamma)$. Then $I(\sigma) \geq 0$, with equality if and only if $\sigma=0$.

REMARK. The condition $C_{\Gamma}<1$ is necessary, since if $C_{\Gamma} \geq 1$ the result in the theorem is contradicted by $I\left(\mu_{e}\right)=V_{\Gamma} \leq 0$.

ProOf. For $\sigma \in \mathcal{S}(\Gamma)$ we may write $\sigma=\sigma(\Gamma) \mu_{e}+\sigma_{0}$, from which it follows that $\sigma_{0}(\Gamma)=0$. A simple argument shows that $\sigma_{0} \in \mathcal{S}(\Gamma)$. 
Then we have, from the definitions of $I(\sigma)$ and $\mu_{e}$,

$$
I(\sigma)=\sigma(\Gamma)^{2} V_{\Gamma}+2 \sigma(\Gamma) \int_{\Gamma} \int_{\Gamma} \log |x-y|^{-1} d \mu_{e}(x) d \sigma_{0}(y)+I\left(\sigma_{0}\right) .
$$

The second term vanishes, since

$$
\begin{aligned}
\int_{\Gamma} \int_{\Gamma} \log |x-y|^{-1} d \mu_{e}(x) d \sigma_{0}(y) & =\int_{\Gamma} U\left(y, \mu_{e}\right) d \sigma_{0}(y) \\
& =V_{\Gamma} \sigma_{0}(\Gamma)=0,
\end{aligned}
$$

where in the second last step we have used Lemma 1.3 and Lemma 1.4.

Thus

$$
I(\sigma)=\sigma(\Gamma)^{2} V_{\Gamma}+I\left(\sigma_{0}\right),
$$

and since $V_{\Gamma}>0$ the result now follows from Lemma 1.5. $\square$

We have already noted that the equilibrium distribution $\mu_{e}$ gives rise to a constant potential (except possibly on a subset of capacity zero) on $\Gamma$. The next result establishes a converse: the only charges with finite energy that can yield a constant potential on $\Gamma$ are the constant multiples of $\mu_{e}$.

THEOREM 1.2. Let $\Gamma$ be a closed bounded set, and assume that $\sigma \in \mathcal{S}(\Gamma)$ is such that

$$
U(x, \sigma)=c=\text { constant }, \quad \text { for } x \in \Gamma,
$$

except on a set of capacity zero. Then $c=\sigma(\Gamma) V_{\Gamma}$, and $\sigma=\sigma(\Gamma) \mu_{e}$.

PROOF. As in the preceding theorem, write $\sigma=\sigma(\Gamma) \mu_{e}+\sigma_{0}$, so that $\sigma_{0}(\Gamma)=0$ and $\sigma_{0} \in \mathcal{S}(\Gamma)$. Then for $x \in \Gamma$ we have

$$
U\left(x, \sigma_{0}\right)=U(x, \sigma)-\sigma(\Gamma) U\left(x, \mu_{e}\right)=c-\sigma(\Gamma) V_{\Gamma},
$$

except on a set of capacity zero. Since the right-hand side is a constant, it follows that

$$
I\left(\sigma_{0}\right)=\int_{\Gamma} U\left(x, \sigma_{0}\right) d \sigma_{0}(x)=\left(c-\sigma(\Gamma) V_{\Gamma}\right) \sigma_{0}(\Gamma)=0 .
$$


Lemma 1.5 now yields $\sigma_{0}=0$ and the result follows.

As a corollary to this result, we now obtain the theorem foreshadowed above, which effectively establishes the uniqueness of the solution of (1).

THEOREM 1.3. (uniqueness). Let $\Gamma$ be a closed bounded set for which the transfinite diameter satisfies $C_{\Gamma} \frac{1}{\tau} 1$. Assume also that $\sigma \in \mathcal{S}(\Gamma)$ is such that

$$
U(x, \sigma)=0, \text { for } x \in \Gamma,
$$

except on a set of capacity zero. Then $\sigma=0$.

Proof. Setting $c=0$ in the preceding result, we obtain $\sigma=\sigma(\Gamma) \mu_{e}$ and $\sigma(\Gamma) V_{\Gamma}=0$. Since $V_{\Gamma} \frac{1}{T} 0$, it follows that $\sigma(\Gamma)=0$, and hence $\sigma=0$.

REMARK 1. The condition $C_{\Gamma} \frac{1}{\tau} 1$ in the theorem is necessary, since if $C_{\Gamma}=1$ the non-zero charge $\mu_{e}$ yields the zero potential except on a set of capacity zero.

REMARK 2. An alternative approach to a uniqueness proof is indicated in [41]. Use Theorem 1.1 to establish uniqueness for the case $C_{\Gamma}<1$ and then use scaling arguments to establish the result for arbitrary $C_{\Gamma} \frac{1}{\tau}$. The present approach is more direct.

REMARK 3. The uniqueness result of $[\mathbf{1 9}]$ requires $\Gamma$ to be a rectifiable and simple closed Jordan curve. Theorem 1.3, however, extends the result to any closed bounded set, including open arcs, boundaries of multiply connected regions and curves which intersect themselves (i.e., have multiple points).

REMARK 4. If $\Gamma$ is a piecewise smooth contour with a finite length, and if for $g \in L_{p}(\Gamma)$ with $p>1$ we let

$$
\sigma(x)=\int_{x_{0}}^{x} g(\tau) d \tau,
$$


where $\int_{x_{0}}^{x}$ denotes an integration along $\Gamma$ from $x_{0}$ to $x$, so that $d \sigma(x)=g(x) d x$, then we automatically have $\sigma \in \mathcal{S}(\Gamma)$. Thus we obtain,

COROLlARY 1.1. Let $\Gamma$ be a piecewise smooth contour with a finite length and transfinite diameter $C_{\Gamma} \frac{\perp}{\tau}$. Assume that $g \in L_{p}(\Gamma)$ with $p>1$ is such that

$$
\int_{\Gamma} \log |x-y|^{-1} g(y) d y=0, \quad \text { for } \quad x \in \Gamma .
$$

Then $g=0$.

2. Sobolev spaces $\mathbf{H}^{\mathbf{t}}$ and $\mathbf{H}_{\mathbf{e}}^{\mathbf{t}}$. The subsequent analysis is performed in Sobolev spaces $H^{t}$ and $H_{e}^{t}$ defined by Fourier series. Our concern in this section is to introduce these Sobolev spaces and establish some useful properties. The Sobolev spaces in the first subsection are the standard ones used for a circular contour. In the second subsection we introduce closely related spaces which will prove useful for a new analysis of open arcs.

2.1. The periodic case. We consider the space of $r$-times continuously differentiable periodic functions on $\mathbf{R}$,

$$
C^{r}(2 \pi)=\left\{f \in C^{r}(\mathbf{R}): f(x+2 \pi)=f(x), \quad x \in \mathbf{R}\right\}, \quad r=0,1, \ldots,
$$

with $C^{\infty}(2 \pi)=\cap_{r} C^{r}(2 \pi)$.

For each $v \in C^{\infty}(2 \pi)$, its Fourier expansion has the form

$$
v(s)=\frac{1}{\sqrt{2 \pi}} \sum_{-\infty}^{\infty} \hat{v}(m) \exp (i m s)
$$

with

$$
\hat{v}(m)=\frac{1}{\sqrt{2 \pi}} \int_{-\pi}^{\pi} v(s) \exp (-i m s) d s, \quad m=0, \pm 1, \pm 2, \ldots .
$$

We define a norm ||$_{t}$ on $C^{\infty}(2 \pi)$ for each real number $t \in \mathbf{R}$ by

$$
|v|_{t}^{2}=\sum_{m \frac{1}{\tau} 0}|m|^{2 t}|\hat{v}(m)|^{2}+|\hat{v}(0)|^{2}
$$


Now the Sobolev space $H^{t}(2 \pi)$ is defined to be the completion of $C^{\infty}(2 \pi)$ with respect to the norm ||$_{t}$. Some useful standard properties are collected in the following theorems and propositions.

THEOREM 2.1.

(i) $H^{t}$ is a Hilbert space with inner product

$$
(u, v)_{t}=\sum_{m \neq 0}|m|^{2 t} \hat{u}(m) \overline{\hat{v}(m)}+\hat{u}(0) \overline{\hat{v}(0)}
$$

(ii) If $\bar{s}<t$, then $H^{s} \supset H^{t}$ and the inclusion is a compact map;

(iii) The dual of $H^{t+\alpha}$, with respect to the inner product $(,)_{t}$, is $H^{t-\alpha}$, i.e., the functional $u \rightarrow(u, v)_{t}$ is bounded on $H^{t+\alpha}$ if and only if $v \in H^{t-\alpha}$, and

$$
|v|_{t-\alpha}=\sup \left\{(u, v)_{t} /|u|_{t+\alpha}: u \in H^{t+\alpha}\right\}
$$

(iv) Interpolation inequality: if $t=(1-\theta) t_{1}+\theta t_{2}, 0 \leq \theta \leq 1$, then

$$
|u|_{t} \leq|u|_{t_{1}}^{1-\theta}|u|_{t_{2}}^{\theta}
$$

These results can be found in $[\mathbf{3 2}, \mathbf{9}, \mathbf{2}]$. Obviously $H^{0}(2 \pi)=L_{2}(2 \pi)$ and $(u, v)_{0}=\int_{-\pi}^{\pi} u(t) \overline{v(t)} d t$. More generally, for $r$ a positive integer $H^{r}$ is equivalent to a Sobolev space $W^{r, 2}$ defined, as in [1], as the space of functions whose generalized derivatives of orders $0,1, \ldots, r$ belong to $L_{2}$.

Proposition 2.1. When $r$ is a positive integer, $H^{r}(2 \pi)$ is equivalent to $W^{r, 2}(2 \pi)=\left\{u \in W^{r, 2}(-\pi, \pi): D^{l} u(-\pi)=D^{l} u(\pi)\right.$, for $l=$ $0, \ldots, r-1\}$.

The proof can be found, for example, in [33].

Now let "->" designate the imbedding map [1]. Then the following imbedding theorems hold:

Proposition 2.2. For $t>\frac{1}{2}, H^{t}(2 \pi)->C^{0}(2 \pi)$. Thus any $u \in H^{t}(2 \pi)$ with $t>\frac{1}{2}$ is a continuous $2 \pi$-periodic function. Moreover the imbedding is a compact map. 
This result follows, for example, from $[\mathbf{3 3}]$ and (ii) in Theorem 2.1 .

\section{Proposition 2.3. (Hardy and Littlewood theorem)}

(i) $H^{1 / 2-1 / p}(2 \pi)->L_{p}(2 \pi)$, for $2 \leq p<+\infty$;

(ii) $L_{p}(2 \pi)->H^{1 / 2-1 / p}(2 \pi)$, for $1<p \leq 2$;

and the converses of (i) and (ii) are not true $[\mathbf{4 8 , 2 2}]$.

2.2. The periodic and even case. In particular, we consider the even periodic function spaces,

$$
C_{e}^{r}(2 \pi)=\left\{f \in C^{r}(2 \pi): f(x)=f(-x), x \in \mathbf{R}\right\}, \quad r=0,1, \ldots,
$$

with $C_{e}^{\infty}(2 \pi)=\cap_{r} C_{e}^{r}(2 \pi)$, which are subspaces of the corresponding spaces in the preceding subsection.

For each $v \in C_{e}^{\infty}(2 \pi)$, its Fourier expansion becomes

$$
v(s)=\sqrt{2 / \pi}\left(\sum_{1}^{\infty} \hat{v}(m) \cos m s+\frac{1}{2} \hat{v}(0)\right),
$$

where $\hat{v}(m)=\sqrt{2 / \pi} \int_{0}^{\pi} v(\sigma) \cos m \sigma d \sigma, m=0,1, \ldots$.

For simplicity we adopt the same norm symbol ||$_{t}$ as before for our new norm

$$
|v|_{t}^{2}=2 \sum_{m=1}^{\infty} m^{2 t}|\hat{v}(m)|^{2}+|\hat{v}(0)|^{2} .
$$

Actually, for $v \in C_{e}^{\infty}(2 \pi)$ the norms defined by (7) and (6) are equal.

The Sobolev space $H_{e}^{t}(2 \pi)$ is defined to be the completion of $C_{e}^{\infty}(2 \pi)$ with respect to norm ||$_{t}$. Similarly to $H^{t}(2 \pi), H_{e}^{t}(2 \pi)$ has all of the properties in Theorem 2.1 if the inner product is defined in the obvious way, and satisfies

Proposition $2.1^{*}$. When $r$ is a positive integer, $H_{e}^{r}(2 \pi)$ is equivalent to $W_{e}^{r, 2}(2 \pi)=\left\{u \in W^{r, 2}(2 \pi): u(x)=u(-x)\right.$, for $\left.x \in \mathbf{R}\right\}$.

Proposition $2.2^{*}$. For $t>\frac{1}{2}, H_{e}^{t}(2 \pi)->C_{e}^{0}(2 \pi)$, and the imbedding is a compact map. 
In fact $H_{e}^{t}(2 \pi)$ is a closed subspace of $H^{t}(2 \pi)$ which consists of even functions. Therefore the results above follow in a straightforward way from Propositions 2.1 and 2.2 .

3. Isometry operators from $\mathbf{H}^{\mathbf{t}}$ to $\mathbf{H}^{\mathbf{t}+\mathbf{1}}, \mathbf{H}_{\mathbf{e}}^{\mathbf{t}}$ to $H_{e}^{t+1}$. Here we study particular integral operators with logarithmic kernels and show that they are isometries from $H^{t}$ to $H^{t+1}$ or from $H_{e}^{t}$ to $H_{e}^{t+1}$. The first case is to some extent known, but the closely related second case seems to be new. It will lead, in the next section, to a novel treatment of open arcs.

3.1 The periodic case. Since to any $v \in H^{0}(2 \pi)$ we have (for example, see $[\mathbf{9}])$

$$
-\frac{1}{\pi} \int_{-\pi}^{\pi} \log \left|2 \sin \frac{s-\sigma}{2}\right| v(\sigma) d \sigma=\frac{1}{\sqrt{2 \pi}} \sum_{|m|>0} \frac{\hat{v}(m)}{|m|} \exp (i m s),
$$

it follows that

$$
\begin{aligned}
-\frac{1}{\pi} & \int_{-\pi}^{\pi} \log \left|2 e^{-\frac{1}{2}} \sin \frac{s-\sigma}{2}\right| v(\sigma) d \sigma \\
& =\frac{1}{\sqrt{2 \pi}}\left(\sum_{|m|>0} \frac{\hat{v}(m)}{|m|} \exp (i m s)+\hat{v}(0)\right) .
\end{aligned}
$$

We let $\Lambda(s-\sigma)=-\frac{1}{\pi} \log \left|2 e^{-\frac{1}{2}} \sin \frac{s-\sigma}{2}\right|$, and $A v(s)=(v(.), \Lambda(s-.))_{0}$. Then (8) shows that

$$
A v(s)=\frac{1}{\sqrt{2 \pi}}\left(\sum_{|m|>0} \frac{\hat{v}(m)}{|m|} \exp (i m s)+\hat{v}(0)\right)
$$

Thus by a simple calculation, we find for $v \in H^{0}$ that $A v \in H^{1}$, $|A v|_{1}=|v|_{0}$ and $(A v, u)_{0}=(v, u)_{-1 / 2}$. Use (9) to extend the domain of definition of $A$ to $H^{t}$, where $t$ is real number. Then we find

$$
\begin{gathered}
|A v|_{t+1}=|v|_{t} \\
(A v, u)_{0}=(v, u)_{-1 / 2}, \quad \text { if } u \in H^{-t-1}, v \in H^{t} .
\end{gathered}
$$


At the same time,

$$
A^{-1} v=\frac{1}{\sqrt{2 \pi}}\left(\sum_{|m|>0}|m| \hat{v}(m) \exp (i m s)+\hat{v}(0)\right), \quad v \in H^{t+1} .
$$

Therefore the operator $A$ is an isometry operator from $H^{t}$ to $H^{t+1}$ for any real number $t$.

Now consider $A^{-1}: H^{t+1} \rightarrow H^{t}$. When $t \geq 0$, we find by a simple calculation

$$
A^{-1} v=-D^{2} A v+J v=-D A D v+J v, \quad v \in H^{t+1},
$$

where $J v=(1 / \sqrt{2 \pi}) \hat{v}(0)$ and $D v(s)=v^{\prime}(s)$. Let $H=D A$. Then

$$
A^{-1}=-D H+J=-H D+J
$$

and $H$ is the well-known Hilbert singular integral operator $[\mathbf{3 4}, \mathbf{3 6}$, 40],

$$
H v(s)=-\frac{1}{2 \pi} p \cdot v \cdot \int_{-\pi}^{\pi} \cot \frac{s-\sigma}{2} v(\sigma) d \sigma,
$$

with the property $H^{2}=-I+J$ and $|H|_{0}=\|H\|_{L_{2}(2 \pi)}=1$.

3.2 The periodic and even case. In parallel we consider the case of $H_{e}^{t}$. To any $v \in H_{e}^{0}(2 \pi)$, we find, for example from [37], that

$-\frac{1}{\pi} \int_{0}^{\pi} \log |\cos s-\cos \sigma| v(\sigma) d \sigma=\sqrt{\frac{2}{\pi}}\left(\sum_{1}^{\infty} \frac{\hat{v}(m)}{m} \cos m s+\frac{\log 2}{2} \hat{v}(0)\right)$.

Thus

$$
\begin{aligned}
& -\frac{1}{\pi} \int_{0}^{\pi} \log \left(2 e^{-1}|\cos s-\cos \sigma|\right) v(\sigma) d \sigma \\
& =\sqrt{\frac{2}{\pi}}\left(\sum_{m=1}^{\infty} \frac{\hat{v}(m)}{m} \cos m s+\frac{1}{2} \hat{v}(0)\right) .
\end{aligned}
$$

Let $\Lambda_{e}(s, \sigma)=-\frac{1}{\pi} \log \left(2 e^{-1}|\cos s-\cos \sigma|\right), A_{e} v(s)=\int_{0}^{\pi} \Lambda_{e}(s, \sigma) v(\sigma) d \sigma$. Then (11) shows that

$$
A_{e} v=\sqrt{\frac{2}{\pi}}\left(\sum_{m=1}^{\infty} \frac{\hat{v}(m)}{m} \cos m s+\frac{1}{2} \hat{v}(0)\right) .
$$


Similarly to $A, A_{e}$ is an isometry operator from $H_{e}^{t}$ to $H_{e}^{t+1}$ for any real number $t$, and

$$
\begin{gathered}
\left|A_{e} v\right|_{t+1}=|v|_{t}, \\
\left(A_{e} v, u\right)_{0}=(v, u)_{-1 / 2}, \quad \text { if } u \in H_{e}^{-t-1}, v \in H_{e}^{t} .
\end{gathered}
$$

A useful and easily verified fact is that

$$
A v=A_{e} v, \quad v \in H_{e}^{0}(2 \pi) .
$$

4. Theoretical analysis for smooth $\Gamma$ (closed or open). Now consider the theoretical analysis of equation (1), and set up the existence-uniqueness theorems of the solutions for a smooth closed contour $\Gamma_{c}$ and smooth open contour $\Gamma_{o}$. The result on the closed contour case is known, but the same simple approach will lead to a natural treatment for the open contour case.

4.1. The closed contour case. Let $\Gamma_{c}$ be a closed and smooth simple curve. Thus there exists a smooth function $v: \mathbf{R} / 2 \pi \mathbf{Z} \rightarrow \Gamma_{c} \subset \mathbf{R}^{2}$, with $\left|v^{\prime}(s)\right| \geq \rho>0$. In this way, we obtain a transformed form of equation (1) on $[-\pi, \pi]$,

$$
\begin{gathered}
-\frac{1}{\pi} \int_{-\pi}^{\pi} \log |v(s)-v(\sigma)|\left|v^{\prime}(\sigma)\right| g(v(\sigma)) d \sigma=\frac{1}{\pi} f(v(s)), \\
s \in[-\pi, \pi] .
\end{gathered}
$$

Let $w(s)=\left|v^{\prime}(s)\right| g(v(s))$ and $\bar{f}(s)=\frac{1}{\pi} f(v(s))$. Then $w(s)$ and $\bar{f}(s)$ are $2 \pi$-periodic functions and

$$
-\frac{1}{\pi} \int_{-\pi}^{\pi} \log |v(s)-v(\sigma)| w(\sigma) d \sigma=\bar{f}(s), \quad s \in[-\pi, \pi] .
$$

In this equation the kernel function is $k(s, \sigma)=-(1 / \pi) \log |v(s)-v(\sigma)|$. Let

$$
K w(s)=(w(.), k(s, .))_{0}=\int_{-\pi}^{\pi} k(s, \sigma) w(\sigma) d \sigma .
$$

Then this equation can be written in the form of an operator equation,

$$
K w=\bar{f} .
$$


Making use of the isometry operator in $\S 3$, we have

$$
A w+(K-A) w=\bar{f},
$$

or

$$
A w+B w=\bar{f}
$$

where

$$
\begin{gathered}
B w(s)=(w(.), b(s, .))_{0}, \\
b(s, \sigma)= \begin{cases}-\frac{1}{\pi} \log \mid e^{\frac{1}{2}\left(\frac{v(s)-v(\sigma)}{2 \sin \frac{s-\sigma}{2}} \mid,\right.}, \quad s-\sigma \in 2 \pi \mathbf{Z}, \\
-\frac{1}{\pi} \log \left|e^{\frac{1}{2}} v^{\prime}(s)\right|, & s-\sigma \in 2 \pi \mathbf{Z} .\end{cases}
\end{gathered}
$$

The function $b(s, \sigma)$ is better behaved than $k(s, \sigma)$. This can be given as a proposition.

Proposition 4.1. $b(s, \sigma)$ is a smooth function of $(s, \sigma)$ on $\mathbf{R}^{2}$ and is $2 \pi$-periodic with respect to each variable.

Since the kernel function $b(s, \sigma)$ is smooth and $2 \pi$-periodic, then for any $w \in H^{t}$, with $t \in \mathbf{R}, B w(s)$ exists and has derivatives of all orders. By a simple argument,

$$
D^{r} B w(s)=\left(w(.), \frac{\partial^{r}}{\partial s^{r}} b(s, .)\right)_{0}, \quad r=0,1,2, \ldots .
$$

Hence $B: H^{t} \rightarrow H^{t+r}$ is bounded for any $t \in \mathbf{R}, r \in \mathbf{Z}^{+}$, so that $B: H^{t} \rightarrow H^{t+1}$ is compact. Thus we have:

Proposition 4.2. $B: H^{t} \rightarrow H^{t+r}$ is bounded for any $t \in \mathbf{R}, r \in \mathbf{Z}^{+}$, and $B: H^{t} \rightarrow H^{t+1}$ is compact for any $t \in \mathbf{R}$.

Let $M=A^{-1} B$. Then $M$ is a compact operator on $H^{t}$, and

$$
K=A+B=A(I+M) .
$$


THEOREM 4.1. If $\Gamma_{c}$ is smooth and simple with $C_{\Gamma_{c}} \frac{1}{T} 1$, then $K: H^{t} \rightarrow H^{t+1}$ is $1-1$ and onto for any $t \in \mathbf{R}$.

Proof. Suppose $w \in H^{t}$ is such that $K w=0$. Then from (14) we have $(I+M) w=0$ and $w=-M w$. According to Proposition 4.2, $w=-M w=-A^{-1} B w$ is a $C^{\infty}(2 \pi)$ function, so that $K w(s) \equiv 0$. In the light of the relation between (1) and (13), we now have

$$
-\frac{1}{\pi} \int_{\Gamma_{c}} \log |x-y| w\left(v^{-1}(y)\right)\left|\left(v^{-1}\right)^{\prime}(y)\right| d y=0, \quad x \in \Gamma_{c},
$$

where $\left(v^{-1}\right)^{\prime}$ denotes the tangential derivative of $v^{-1}$. Since $w(s)$ is smooth, $g(x)=w\left(v^{-1}(x)\right)\left|\left(v^{-1}\right)^{\prime}(x)\right| \in L_{2}\left(\Gamma_{c}\right)$. By use of Corollary 1.1 we have $g=0$ in $L_{2}\left(\Gamma_{c}\right)$, so that $w(s) \equiv 0$. This means that $K: H^{t} \rightarrow H^{t+1}$, is $1-1$ for $t \in \mathbf{R}$.

It follows that the operator $I+M: H^{t} \rightarrow H^{t}$ is $1-1$ and according to the standard Fredholm theory is onto. This implies that $K: H^{t} \rightarrow$ $H^{t+1}$ is onto. Since $K$ is both 1-1 and onto, the proof is now complete.

4.2. The open contour case. Now we discuss equation (1) for the case of an open and smooth simple curve $\Gamma_{o}$. In this case we adopt a different parametric representation of the curve.

Let $v(s)$ be a smooth parametrization of the form, $v:[-1,1] \rightarrow \Gamma_{o} \subset$ $\mathbf{R}^{2}$, with $\left|v^{\prime}(s)\right| \geq \rho>0$. Then define $a:[0, \pi] \rightarrow \Gamma_{o}$ by $a(t)=v(\cos t)$, so that $\Gamma_{o}$ is now considered to be parametrized by $t=\cos ^{-1} s$. In this way, we have a transformed form of equation (1) on $[0, \pi]$,

$$
\begin{aligned}
& -\frac{1}{\pi} \int_{0}^{\pi} \log |a(t)-a(\tau)|\left|v^{\prime}(\cos \tau)\right| \sin \tau g(a(\tau) d \tau \\
& =\frac{1}{\pi} f(a(t)), \quad t \in[0, \pi] .
\end{aligned}
$$

Let $w(t)=\left|v^{\prime}(\cos t)\right||\sin t| g(a(t)), \bar{f}(t)=\frac{1}{\pi} f(a(t))$. Then $w(t)$ and $\bar{f}(t)$ are even $2 \pi$-periodic functions on $\mathbf{R}$. The integral equation, which we now write as

$$
-\frac{1}{\pi} \int_{0}^{\pi} \log |a(t)-a(\tau)| w(\tau) d \tau=\bar{f}(t), \quad t \in[-\pi, \pi],
$$


can be thought of as mapping one even $2 \pi$-periodic function into another. In this equation the kernel function is $k_{e}(t, \tau)=-(1 / \pi) \log \mid a(t)-$ $a(\tau) \mid$. Letting

$$
K_{e} w(t)=\int_{0}^{\pi} k_{e}(t, \tau) w(\tau) d \tau, \quad t \in[-\pi, \pi],
$$

it then can be written as an operator equation

$$
K_{e} w=\bar{f} .
$$

Making use of the isometry operator in $\S 3$, we have

$$
A_{e} w+\left(K_{e}-A_{e}\right) w=\bar{f},
$$

or

$$
A_{e} w+B_{e} w=\bar{f},
$$

where $B_{e} w(t)=\int_{0}^{\pi} b_{e}(t, \tau) w(\tau) d \tau$ and

$$
b_{e}(t, \tau)= \begin{cases}-\frac{1}{\pi} \log \left|\frac{e}{2} \frac{a(t)-a(\tau)}{\cos t-\cos \tau}\right|, & t-\tau \text { and } t+\tau \in 2 \pi \mathbf{Z}, \\ -\frac{1}{\pi} \log \left|\frac{e}{2} v^{\prime}(\cos t)\right|, & t-\tau \text { or } t+\tau \in 2 \pi \mathbf{Z} .\end{cases}
$$

The function $b_{e}(t, \tau)$ is better behaved than $k_{e}(t, \tau)$.

Proposition 4.3. $b_{e}(t, \tau)$ is a smooth function of $(t, \tau)$ on $\mathbf{R}^{2}$ and is $2 \pi$-periodic and even with respect to each variable.

Similar to the discussion for $\Gamma_{c}$, we can easily find that:

Proposition 4.4. $B_{e}: H_{e}^{t} \rightarrow H_{e}^{t+r}$ is bounded for any $t \in \mathbf{R}, r \in$ $\mathbf{Z}^{+}$and $B_{e}: H_{e}^{t} \rightarrow H_{e}^{t+1}$ is compact for any $t \in \mathbf{R}$.

Now let $M_{e}=\left(A_{e}\right)^{-1} B_{e}$. Then $M_{e}$ is a compact operator on $H_{e}^{t}$ and

$$
K_{e}=A_{e}\left(I+M_{e}\right) .
$$


THEOREM 4.2. If $\Gamma_{o}$ is smooth and simple with $C_{\Gamma_{o}} \frac{1}{\tau} 1$, then $K_{e}: H_{e}^{t} \rightarrow H_{e}^{t+1}$ is $1-1$ and onto for any $t \in \mathbf{R}$.

Proof. Suppose $w \in H_{e}^{t}$ such that $K_{e} w=0$. Then from (17) $\left(I+M_{e}\right) w=0$, so that $w=-M_{e} w$. According to Proposition 4.2, $w=-M_{e} w$ is a $C_{e}^{\infty}(2 \pi)$ function, so $K_{e} w(s) \equiv 0$. In the light of the relation between (1) and (16), we have

$$
-\frac{1}{\pi} \int_{\Gamma_{o}} \log |x-y| w\left(a^{-1}(y)\right) \frac{\left|\left(v^{-1}\right)^{\prime}(y)\right|}{\sin \left(a^{-1}(y)\right)} d y=0, \quad x \in \Gamma_{o} .
$$

By a simple calculation

$$
\frac{1}{\sin \left(a^{-1}(x)\right)}=\frac{1}{\sqrt{1-\left(v^{-1}(x)\right)^{2}}}
$$

which is an integrable function on $\Gamma_{o}$, having the singularity $\left|x-x_{i}\right|^{-1 / 2}$ at the end points $x_{i}(i=1,2)$ of $\Gamma_{o}$, where $x_{1}=a(0), x_{2}=a(\pi)$. Since $w(s)$ is smooth, we have

$$
g(x)=\frac{w\left(a^{-1}(x)\right)\left|\left(v^{-1}\right)^{\prime}(x)\right|}{\sin \left(a^{-1}(x)\right)} \in L_{p}\left(\Gamma_{o}\right), \quad \text { with } 1<p<2 .
$$

By use of Corollary 1.1 we find that $g=0$ in $L_{p}\left(\Gamma_{o}\right)$, from which follows $w(s) \equiv 0$. This means that $K_{e}: H_{e}^{t} \rightarrow H_{e}^{t+1}$ is $1-1$. Similarly to the proof of Theorem 4.1, we have that $K_{e}: H_{e}^{t} \rightarrow H_{e}^{t+1}$ is onto as well. As a result $K_{e}$ is $1-1$ and onto.

4.3 Discussion. As a byproduct of the analysis above, the different character of the solution of (1) on closed and open contours can now be understood. For a smooth closed contour $\Gamma_{c}$, when $f(x)$ is $r+1$ times differentiable ( $r$ an integer) along the boundary $\Gamma_{c}$, the solution $g(x)=\left|\left(v^{-1}\right)^{\prime}(x)\right| w\left(v^{-1}(x)\right)$ will be $r$ times differentiable; with $f(x)$ smooth, the solution $g(x)$ will be smooth. But for a smooth open contour $\Gamma_{o}$, when $f(x)$ is smooth along $\Gamma_{o}$ we have

$$
g(x)=\frac{w\left(a^{-1}(x)\right)\left|\left(v^{-1}\right)^{\prime}(x)\right|}{\sqrt{1-\left(v^{-1}(x)\right)^{2}}},
$$


in which (since $w(t)$ is a smooth even function) the factor $w\left(a^{-1}(x)\right)$ is well behaved. Thus $g$ possesses the singular factor $\left(1-\left(v^{-1}(x)\right)^{2}\right)^{-1 / 2}$, unless $w\left(a^{-1}(x)\right)$ vanishes at the appropriate end point of $\Gamma_{o}$.

Examples:

(i) $\Gamma_{c}$ a circle of radius $a, a \frac{1}{\tau} 1$ : the solution of $-\int_{\Gamma_{c}} \log |x-y|$ $\cdot g(y) d y=1$ is the constant function

$$
g(x)=-(a \log a 2 \pi)^{-1}
$$

(ii) $\Gamma_{o}$ an interval $[-a, a], a \frac{1}{\tau} 2$ : the solution of $-\int_{-a}^{a} \log |x-y|$ $g(y) d y=1$ is the singular function

$$
g(x)=-\frac{1}{\pi \log (a / 2)} \frac{1}{\sqrt{a^{2}-x^{2}}} .
$$

4.4. Extension to the equation $\left(1^{*}\right)$. To some extent, the analysis of equation (1) can be extended to equation $\left(1^{*}\right)$. We assume that $F(x, y)$ is smooth along $\Gamma \times \Gamma$. By appropriate changes of variables, we obtain the transformed forms of equation $\left(1^{*}\right)$ corresponding to (13) and (16) respectively:

$$
\begin{aligned}
& (K+F) w=\bar{f}, \text { for } \Gamma_{c} ; \\
& \left(K_{e}+F_{e}\right) w=\bar{f}, \text { for } \Gamma_{o} \text {; }
\end{aligned}
$$

where

$$
\begin{aligned}
& F w(s)=-\frac{1}{\pi} \int_{-\pi}^{\pi} F(v(s), v(\sigma)) w(\sigma) d \sigma, \quad s \in[-\pi, \pi], \\
& F_{e} w(t)=-\frac{1}{\pi} \int_{0}^{\pi} F(a(t), a(\tau)) w(\tau) d \tau, \quad t \in[-\pi, \pi] .
\end{aligned}
$$

Obviously $F: H^{t} \rightarrow H^{t+1}$ and $F_{e}: H_{e}^{t} \rightarrow H_{e}^{t+1}$ are compact for any $t \in \mathbf{R}$. Let $\bar{M}=A^{-1}(B+F), \bar{M}_{e}=A_{e}^{-1}\left(B_{e}+F_{e}\right)$, then $\bar{M}: H^{t} \rightarrow H^{t}, \bar{M}_{e}: H_{e}^{t} \rightarrow H_{e}^{t}$ are compact, and (18) and (19) are equivalent to $\left(18^{*}\right)$ and $\left(19^{*}\right)$ respectively,

$$
\begin{aligned}
(I+\bar{M}) w & =A^{-1} \bar{f}, \\
\left(I+\bar{M}_{e}\right) w & =A_{e}^{-1} \bar{f},
\end{aligned}
$$


which are second kind Fredholm equations. Let $N^{t}(Q)$ and $N_{e}^{t}(Q)$ denote the null space of operator $Q$ on $H^{t}$ and $H_{e}^{t}$ respectively, and $N^{\infty}(Q)=\cap_{t} N^{t}(Q), N_{e}^{\infty}(Q)=\cap_{t} N_{e}^{t}(Q)$. Then according to the standard Fredholm theory, we obtain

THEOREM 4.3. Suppose that a smooth simple $\Gamma$ is parametrized as a smooth function $v(s)$ as before, with $\left|v^{\prime}(s)\right| \geq \rho>0$. If $F(x, y)$ is smooth along $\Gamma \times \Gamma$, then

(i) For any $t \in \mathbf{R}, N^{t}(K+F)=N^{\infty}(K+F)$, and $\operatorname{dim} N^{\infty}(K+$ $F)<+\infty$. Moreover $K+F: H^{t} \rightarrow H^{t+1}$ is 1-1 and onto iff $\operatorname{dim} N^{\infty}(K+F)=0$.

(ii) For any $t \in \mathbf{R}, N_{e}^{t}\left(K_{e}+F_{e}\right)=N_{e}^{\infty}\left(K_{e}+F_{e}\right)$ and $\operatorname{dim} N_{e}^{\infty}\left(K_{e}+\right.$ $\left.F_{e}\right)<+\infty$. Moreover $K_{e}+F_{e}: H_{e}^{t} \rightarrow H_{e}^{t+1}$ is 1-1 and onto iff $\operatorname{dim} N_{e}^{\infty}\left(K_{e}+F_{e}\right)=0$.

With less restriction on $\Gamma$, we have the following weaker results, which will be discussed in the last section.

THEOREM 4.4 .

(i) For $\Gamma_{c}$, suppose that $v^{\prime}(s)$ is continuous with $\left|v^{\prime}(s)\right| \geq \rho>0$ and $\frac{\partial}{\partial s} F(v(s), v(\sigma))$ is continuous on $[-\pi, \pi] \times[-\pi, \pi]$. Then $\operatorname{dim} N^{0}(K+$ $F)<+\infty$. Moreover $K+F: H^{0} \rightarrow H^{1}$ is 1-1 and onto iff $\operatorname{dim} N^{0}(K+F)=0$;

(ii) For $\Gamma_{o}$, suppose that $a^{\prime}(t)$ is continuous with $\left|a^{\prime}(t)\right| \geq \rho|\sin t|, \rho>$ 0 and $\frac{\partial}{\partial t} F(a(t), a(\tau))$ continuous on $[-\pi, \pi] \times[0, \pi]$. Then $\operatorname{dim} N_{e}^{0}\left(K_{e}+\right.$ $\left.F_{e}\right)<+\infty$. Moreover $K_{e}+F_{e}: H_{e}^{0} \rightarrow H_{e}^{1}$ is 1-1 and onto iff $\operatorname{dim} N_{e}^{0}\left(K_{e}+F_{e}\right)=0$.

5. Analysis for polygonal $\boldsymbol{\Gamma}_{c}$. When $\Gamma_{c}$ is a polygon, the discussion of equation (1) is complicated by the fact that the solution of equation (1) in general has singularities at the corners $[\mathbf{3 0}, \mathbf{2 0}, \mathbf{2 9}$, $12,21,41,47]$. As in $\S 4.1$, we parametrize the curve by a function $v: \mathbf{R} / 2 \pi \mathbf{Z} \rightarrow \Gamma_{c}$, but now $v$ is only piecewise smooth and satisfies $\left|v^{\prime}(s)\right| \geq \rho>0$ only away from the corners. Thus the integral equation is again $\left(12^{*}\right)$ or $(13)$, but because of the lack of smoothness of the kernel we must now restrict the operator $K$ in (13) to the space $H^{0}$, 
rather than to the more general space $H^{t}$. We can no longer expect to decompose the operator $K: H^{0} \rightarrow H^{1}$ into a sum of an isometry operator and a compact operator as in $\left(13^{*}\right)$. Thus some new techniques have to be introduced.

As we know from $\left(13^{*}\right), K=A+B$. (Note that $B w(s)$ is well defined even if $s$ is a corner point of the polygon.) Now we are concerned with $D B$, which we will show in the next theorem can be written as

$$
D B=R+E,
$$

where $E$ is a compact operator on $H^{0}$ and $R$ is a bounded operator, with $|R|_{0}=\|\left. R\right|_{L_{2}}<1$. By noting that $A^{-1}=-H D+J$ (see $\S 3$ ), we have

$$
\begin{aligned}
K & =A\left(I+A^{-1} B\right)=A(I-H D B+J B) \\
& =A(I-H R-H E+J B) .
\end{aligned}
$$

Let $\bar{E}=-H E+J B$. Then $\bar{E}$ is compact on $H^{0}$ and $|H R|_{0}=$ $\|H R\|_{L_{2}} \leq\|H\|_{L_{2}}\|R\|_{L_{2}}=\|R\|_{L_{2}}<1$, so that the operator $I-$ $H R+\bar{E}$ is a Fredholm operator of index 0 on $H^{0}$. Now suppose that $C_{\Gamma_{c}} \pm 1$. From Corollary 1.1 we see that $K: H^{0} \rightarrow H^{1}$ is $1-1$. It follows that $I-H R+\bar{E}: H^{0} \rightarrow H^{0}$ is 1-1. So from the Fredholm theorem it is onto as well. Thus $K: H^{0} \rightarrow H^{1}$ is 1-1 and onto.

In the following we will lay stress on the decomposition (20) of $D B$. A similar approach has been adopted for the second kind of boundary integral equations in [8].

It is convenient to further specialize the function $v(s)$ by taking the parameter proportional to the arc length; that is $\left|v^{\prime}(s)\right|=d\left(\Gamma_{c}\right) / 2 \pi$, where $d\left(\Gamma_{c}\right)$ is the length of $\Gamma_{c}$. Suppose $v(s)=(x(s), y(s))$ and that the corner points are at $v_{1}=v\left(s_{1}\right), \ldots, v_{m}=v\left(s_{m}\right)$, with $-\pi=s_{0}<s_{1}<\ldots<s_{m}<s_{m+1}=\pi, v_{0}=v\left(s_{0}\right)=v_{m+1}=v\left(s_{m+1}\right)$. The arc-length function $v(s)=(x(s), y(s))$ then can be described as:

$$
v(s)=v_{i}+\frac{s-s_{i}}{s_{i+1}-s_{i}}\left(v_{i+1}-v_{i}\right), \quad \text { for } s_{i} \leq s \leq s_{i+1}, i=0, \ldots, m .
$$

At each $v_{i}$ the number $\chi_{i} \in(-1,1)$ is defined by requiring $\left(1-\chi_{i}\right) \pi$ to be the interior angle $v_{i-1} \hat{v}_{i} v_{i+1}$ for $i=1, \ldots, m$. For the sake of convenience, we let

$$
b=(1 / 2) \min \left\{\left|s_{i}-s_{i-1}\right| ; \quad i=1, \ldots, m+1\right\},
$$




$$
s_{i, \pm 1 / 2}=s_{i} \pm b, \quad i=1, \ldots, m .
$$

Now for $s \frac{1}{T} s_{i}, i=1, \ldots, m$,

$$
\begin{aligned}
D B w(s)= & -\frac{1}{\pi} \int_{-\pi}^{\pi} \frac{\partial}{\partial s} \log \left|e^{\frac{1}{2}} \frac{v(s)-v(\sigma)}{2 \sin \frac{s-\sigma}{2}}\right| w(\sigma) d \sigma \\
= & -\frac{1}{\pi} \int_{-\pi}^{\pi} h(s, \sigma) w(\sigma) d \sigma \\
= & -\frac{1}{\pi} \int_{s_{1,-1 / 2}}^{s_{m, 1 / 2}} h(s, \sigma) w(\sigma) d \sigma \\
& -\frac{1}{\pi}\left(\int_{s_{m, 1 / 2}}^{\pi}+\int_{-\pi}^{s_{1,-1 / 2}}\right) h(s, \sigma) w(\sigma) d \sigma,
\end{aligned}
$$

where

$$
h(s, \sigma)=\frac{x^{\prime}(s)(x(s)-x(\sigma))+y^{\prime}(s)(y(s)-y(\sigma))}{(x(s)-x(\sigma))^{2}+(y(s)-y(\sigma))^{2}}-\frac{1}{2} \cot \frac{s-\sigma}{2} .
$$

Since $v(s)$ is $2 \pi$-periodic and smooth except at the corner points, a simple computation shows that the last two integrals above determine a compact operator on $H^{0}$. The first integral can be written as

$$
\begin{gathered}
-\frac{1}{\pi} \int_{s_{1,-1 / 2}}^{s_{m, 1 / 2}}\left(\frac{x^{\prime}(s)(x(s)-x(\sigma))+y^{\prime}(s)(y(s)-y(\sigma))}{(x(s)-x(\sigma))^{2}+(y(s)-y(\sigma))^{2}}-\frac{1}{s-\sigma}\right) w(\sigma) d \sigma \\
-\frac{1}{\pi} \int_{s_{1,-1 / 2}}^{s_{m, 1 / 2}}\left(\frac{1}{s-\sigma}-\frac{1}{2} \cot \frac{s-\sigma}{2}\right) w(\sigma) d \sigma
\end{gathered}
$$

or

$$
\int_{s_{1,-1 / 2}}^{s_{m, 1 / 2}} G(s, \sigma) w(\sigma) d \sigma+\int_{s_{1,-1 / 2}}^{s_{m, 1 / 2}} r(s-\sigma) w(\sigma) d \sigma,
$$

where $r(s-\sigma)=-\frac{1}{\pi}\left(\frac{1}{s-\sigma}-\frac{1}{2} \cot \frac{s-\sigma}{2}\right)$ is a smooth function of $(s, \sigma) \in$ $[-\pi, \pi] \times\left[s_{1,-1 / 2}, s_{m, 1 / 2}\right]$, which makes the last integral above determine a compact operator. The first integral we write as

$$
\begin{aligned}
\int_{s_{1,-1 / 2}}^{s_{m, 1 / 2}} G(s, \sigma) w(\sigma) d \sigma= & \sum_{i=1}^{m} \int_{s_{i,-1 / 2}}^{s_{i, 1 / 2}} G(s, \sigma) w(\sigma) d \sigma \\
& +\sum_{i=1}^{m-1} \int_{s_{i, 1 / 2}}^{s_{i+1,-1 / 2}} G(s, \sigma) w(\sigma) d \sigma .
\end{aligned}
$$


The second sum above determines a compact operator on $H^{0}$. Moreover, $G(s, \sigma)$ is a smooth function of $(s, \sigma) \in\left([-\pi, \pi] \backslash\left[s_{i}, s_{i, 1 / 2}\right]\right) \times$ $\left[s_{i,-1 / 2}, s_{i}\right]$ and $\left([-\pi, \pi] \backslash\left[s_{i,-1 / 2}, s_{i}\right]\right) \times\left[s_{i}, s_{i, 1 / 2}\right], i=1, \ldots, m$. Thus the first sum above as an operator on $H^{0}$ can be written as

$$
R+(\text { compact part })
$$

where

$$
R w(s)= \begin{cases}\int_{s_{i}}^{s_{i, 1 / 2}} G(s, \sigma) w(\sigma) d \sigma, & s \in\left[s_{i,-1 / 2}, s_{i}\right), i=1, \ldots, m \\ \int_{s_{i,-1 / 2}}^{s_{i}} G(s, \sigma) w(\sigma) d \sigma, & s \in\left(s_{i}, s_{i, 1 / 2}\right], i=1, \ldots, m \\ 0, & \text { otherwise }\end{cases}
$$

And for $(s, \sigma) \in\left(\left(s_{i}, s_{i, 1 / 2}\right] \times\left[s_{i,-1 / 2}, s_{i}\right]\right) \cup\left(\left[s_{i,-1 / 2}, s_{i}\right) \times\left[s_{i}, s_{i+1 / 2}\right]\right)$, by simple calculation, we have

$$
\begin{aligned}
G(s, \sigma) & =\frac{1-\cos \chi_{i} \pi}{\pi} \\
& \cdot \frac{\left(\sigma-s_{i}\right)\left(\sigma-s_{i}+s-s_{i}\right)}{(s-\sigma)\left[\left(s-s_{i}\right)^{2}+\left(\sigma-s_{i}\right)^{2}-2\left(s-s_{i}\right)\left(\sigma-s_{i}\right) \cos \chi_{i} \pi\right]}
\end{aligned}
$$

Summing up all the discussions above, we find that

$$
D B=R+E
$$

where $E$ is a compact operator on $H^{0}$.

For the analysis of the operator $R$ we need a lemma.

LEMMA 5.1. For any real number $\chi \in(-1,1)$, if

$$
r_{\chi}(s, \sigma)=\frac{1-\cos \chi \pi}{\pi} \frac{\sigma(\sigma-s)}{(\sigma+s)\left(s^{2}+\sigma^{2}+2 s \sigma \cos \chi \pi\right)},
$$

the integral operator defined by

$$
R_{\chi} w(s)=\int_{0}^{1} r_{\chi}(s, \sigma) w(\sigma) d \sigma, \quad 0<s \leq 1,
$$

is a bounded operator on $L_{2}[0,1]$, with $\left\|R_{\chi}\right\|_{L_{2}[0,1]} \leq 1-\cos \frac{\chi \pi}{2}<1$. 
In order to prove this result we introduce the Mellin transform as follows,

$$
\hat{w}(\lambda)=\int_{0}^{\infty} \sigma^{i \lambda-1} w(\sigma) d \sigma
$$

and describe some of its properties.

Proposition 5.1 (Parseval relation). For $w(\sigma) \in L_{2}(0, \infty)$,

$$
\int_{-\infty}^{\infty}|\hat{w}(\lambda-i / 2)|^{2} d \lambda=2 \pi \int_{0}^{\infty}|w(\sigma)|^{2} d \sigma
$$

In fact with a change of variable $\sigma=e^{-t}$ in (21), we have

$$
\hat{w}(\lambda-i / 2)=\int_{-\infty}^{\infty} e^{-i \lambda t} e^{-t / 2} w\left(e^{-t}\right) d t
$$

which is the Fourier transform of the function $e^{-t / 2} w\left(e^{-t}\right)$. Thus, from the Parseval relation for Fourier transform, follows

$$
\int_{-\infty}^{\infty}|\hat{w}(\lambda-i / 2)|^{2} d \lambda=2 \pi \int_{-\infty}^{\infty}\left|w\left(e^{-t}\right)\right|^{2} e^{-t} d y=2 \pi \int_{0}^{\infty}|w(\sigma)|^{2} d \sigma .
$$

By use of the same change of variables, the Mellin transform of the function

$$
u(s)=\int_{0}^{\infty} k\left(\frac{s}{\sigma}\right) w(\sigma) \frac{d \sigma}{\sigma}
$$

at the point $\lambda-i / 2$ is

$$
\hat{u}(\lambda-i / 2)=\int_{-\infty}^{\infty} e^{-i \lambda t} \int_{-\infty}^{\infty} e^{-(t-\tau) / 2} k\left(e^{-(t-\tau)}\right) e^{-\tau / 2} w\left(e^{-\tau}\right) d \tau d t .
$$

This is the Fourier transform of the convolution of the functions $e^{-t / 2} k\left(e^{-t}\right)$ and $e^{-t / 2} w\left(e^{-t}\right)$. From a corresponding result for Fourier transform equals (see, for example, $[\mathbf{3 5}]$ )

$$
\int_{-\infty}^{\infty} e^{-i \lambda t} e^{-t / 2} k\left(e^{-t}\right) d t \int_{-\infty}^{\infty} e^{-i \lambda t} e^{-t / 2} w\left(e^{-t}\right) d t=\hat{k}(\lambda-i / 2) \hat{w}(\lambda-i / 2),
$$


under the condition $e^{-t / 2} k\left(e^{-t}\right) \in L_{1}(\mathbf{R})$ and $e^{-t / 2} w\left(e^{-t}\right) \in L_{2}(\mathbf{R})$. As a result we have

Proposition 5.2 (convolution theorem). For

$$
u(s)=\int_{0}^{\infty} k\left(\frac{s}{\sigma}\right) w(\sigma) \frac{d \sigma}{\sigma},
$$

if $w(\sigma) \in L_{2}(0, \infty)$ and $\sigma^{-1 / 2} k(\sigma) \in L_{1}(0, \infty)$, then

$$
\hat{u}(\lambda-i / 2)=\hat{k}(\lambda-i / 2) \hat{w}(\lambda-i / 2),
$$

in $L_{2}(\mathbf{R})$. (See also $\left.[\mathbf{4 2}, \mathbf{1 3}]\right)$.

Proof OF Lemma 5.1: Let $u(s)=R_{\chi} w(s)$. Then $u(s)=R_{\chi} w_{0}(s)$, where

$$
w_{0}(s)= \begin{cases}w(s), & s \in[0,1] \\ 0, & s \in(1, \infty) .\end{cases}
$$

And

$$
\begin{aligned}
R_{\chi} w_{0}(s) & =\int_{0}^{\infty} \frac{1-\cos \chi \pi}{\pi} \frac{\left(1-\frac{s}{\sigma}\right) w_{0}(\sigma)}{\left(1+\frac{s}{\sigma}\right)\left(1+2 \frac{s}{\sigma} \cos \chi \pi+\left(\frac{s}{\sigma}\right)^{2}\right)} \frac{d \sigma}{\sigma} \\
& =\int_{0}^{\infty} k_{\chi}\left(\frac{s}{\sigma}\right) w_{0}(\sigma) \frac{d \sigma}{\sigma},
\end{aligned}
$$

where

$$
k_{\chi}(\tau)=\frac{1-\cos \chi \pi}{\pi} \frac{1-\tau}{(1+\tau)\left(1+2 \tau \cos \chi \pi+\tau^{2}\right)} .
$$


Now, by use of (22) and (23),

$$
\begin{aligned}
\int_{0}^{1}|u(\sigma)|^{2} d \sigma & \leq \int_{0}^{\infty}|u(\sigma)|^{2} d \sigma=(2 \pi)^{-1} \int_{-\infty}^{\infty}|\hat{u}(\lambda-i / 2)|^{2} d \lambda \\
& =(2 \pi)^{-1} \int_{-\infty}^{\infty}\left|\hat{k}_{\chi}(\lambda-i / 2)\right|^{2}\left|\hat{w}_{0}(\lambda-i / 2)\right|^{2} d \lambda \\
& \leq \sup _{\lambda \in \mathbf{R}}\left|\hat{k}_{\chi}(\lambda-i / 2)\right|^{2}(2 \pi)^{-1} \int_{-\infty}^{\infty}\left|\hat{w}_{0}(\lambda-i / 2)\right|^{2} d \lambda \\
& =\sup _{\lambda \in \mathbf{R}}\left|\hat{k}_{\chi}(\lambda-i / 2)\right|^{2} \int_{0}^{\infty}\left|w_{0}(\sigma)\right|^{2} d \sigma \\
& =\sup _{\lambda \in \mathbf{R}}\left|\hat{k}_{\chi}(\lambda-i / 2)\right|^{2} \int_{0}^{1}|w(\sigma)|^{2} d \sigma,
\end{aligned}
$$

where

$$
\hat{k}_{\chi}(\lambda-i / 2)=\frac{1-\cos (1 / 2-i \lambda) \chi \pi}{\cosh \lambda \pi} .
$$

Thus the lemma follows because

$$
\begin{aligned}
\sup _{\lambda \in \mathbf{R}}\left|\hat{k}_{\chi}(\lambda-i / 2)\right| & =\sup _{\lambda \in \mathbf{R}}\left|\frac{\cosh \lambda \chi \pi-\cos \chi \pi / 2}{\cosh \lambda \pi}\right| \\
& =1-\cos \chi \pi / 2, \quad \text { for } \chi \in(-1,1) . \square
\end{aligned}
$$

TheOREM 5.1 For a polygonal $\Gamma_{c}$, write $K=A+B$ as in $\left(13^{*}\right)$. Then $D B=R+E$, where $E$ is compact on $H^{0}$ and

$$
\|R\|_{L_{2}(2 \pi)} \leq \max _{1 \leq i \leq m}\left(1-\cos \chi_{i} \pi / 2\right)<1 .
$$

ProOF:

$$
\begin{aligned}
\|R w\|_{L_{2}(2 \pi)}^{2} & =\|R w\|_{L_{2}[-\pi, \pi]}^{2} \\
& =\sum_{i=1}^{m}\left(\|R w\|_{L_{2}\left[s_{i,-1 / 2}, s_{i}\right]}^{2}+\|R w\|_{L_{2}\left[s_{i}, s_{i, 1 / 2}\right]}^{2}\right) .
\end{aligned}
$$


Now

$$
\begin{aligned}
& \|R w\|_{L_{2}\left[s_{i,-1 / 2} \cdot s_{i}\right]}^{2} \int_{s_{i, 1 / 2}}^{s_{i}}\left(\int_{s_{i}}^{s_{i, 1 / 2}} \frac{1-\cos \chi_{i} \pi}{\pi}\right. \\
& \left.\cdot \frac{\left(\sigma-s_{i}\right)\left(\sigma-s_{i}+s-s_{i}\right) w(\sigma) d \sigma}{(s-\sigma)\left[\left(s-s_{i}\right)^{2}+\left(\sigma-s_{i}\right)^{2}-2\left(s-s_{i}\right)\left(\sigma-s_{i}\right) \cos \chi_{i} \pi\right]}\right)^{2} d s \\
& =\int_{0}^{b}\left(\int_{0}^{b} \frac{1-\cos \chi_{i} \pi}{\pi} \frac{\sigma(\sigma-s) w\left(\sigma+s_{i}\right) d \sigma}{(s+\sigma)\left(s^{2}+\sigma^{2}+2 s \sigma \cos \chi_{i} \pi\right)}\right)^{2} d s \\
& =b \int_{0}^{1}\left(\int_{0}^{1} \frac{1-\cos \chi_{i} \pi}{\pi} \frac{\sigma(\sigma-s) w\left(b \sigma+s_{i}\right) d \sigma}{(s+\sigma)\left(s^{2}+\sigma^{2}+2 s \sigma \cos \chi_{i} \pi\right)}\right)^{2} d s .
\end{aligned}
$$

By using Lemma 5.1, we obtain

$$
\begin{aligned}
\|R w\|_{L_{2}\left[s_{i,-1 / 2}, s_{i}\right]}^{2} & \leq\left\|R_{\chi_{i}}\right\|_{L_{2}[0,1]}^{2} b \int_{0}^{1} w^{2}\left(b \sigma+s_{i}\right) d \sigma \\
& =\left\|R_{\chi_{i}}\right\|_{L_{2}[0,1]}^{2} \int_{s_{i}}^{s_{i, 1 / 2}} w^{2}(\sigma) d \sigma .
\end{aligned}
$$

Similarly, we have

$$
\|R w\|_{L_{2}\left[s_{i}, s_{i, 1 / 2}\right]}^{2} \leq\left\|R_{\chi_{i}}\right\|_{L_{2}[0,1]}^{2} \int_{s_{i,-1 / 2}}^{s_{i}} w^{2}(\sigma) d \sigma .
$$

Therefore

$$
\begin{aligned}
\|R w\|_{L_{2}[-\pi, \pi]}^{2} \leq & \left(\max _{1 \leq i \leq m}\left\|R_{\chi_{i}}\right\|_{L_{2}[0,1]}\right)^{2} \\
& \sum_{i=1}^{m}\left(\int_{s_{i,-1 / 2}}^{s_{i}} w^{2}(\sigma) d \sigma+\int_{s_{i}}^{s_{i, 1 / 2}} w^{2}(\sigma) d \sigma\right) \\
\leq & \left(\max _{1 \leq i \leq m}\left\|R_{\chi_{i}}\right\|_{L_{2}[0,1]}\right)^{2}\|w\|_{L_{2}[-\pi, \pi]}^{2} .
\end{aligned}
$$

Thus

$$
\|R\|_{L_{2}(2 \pi)} \leq \max _{1 \leq i \leq m}\left\|R_{\chi_{i}}\right\|_{L_{2}[0,1]} \leq \max _{1 \leq i \leq m}\left(1-\cos \chi_{i} \pi / 2\right)<1 .
$$


As a result of the discussion in the beginning of this section, we obtain

THEOREM 5.2. For a polygonal $\Gamma_{c}$, if $C_{\Gamma_{c}} \frac{1}{\tau} 1$, then $K: H^{0} \rightarrow H^{1}$ is 1-1 and onto.

REMARK. The above proof of this theorem is simpler than that of [12]. The analysis enables us to view the integral equation of the first kind (13),

$$
K w=\bar{f},
$$

as a Fredholm equation of the second kind

$$
(I-H R+\bar{E}) w=f^{*},
$$

where $f^{*}=A^{-1} \bar{f}, \bar{E}$ is compact and $H R$ is bounded on $H^{0}$ with $|H R|_{0}<1$. This will lead to a numerical analysis of the Fredholm equation of the second kind when numerical methods for the integral equation of the first kind (1) are considered.

From the discussion of the decomposition of $D B$, we can find that if $v^{\prime}(s)$ is continuous, the kernel function of $D B$ will be continuous on $[-\pi, \pi] \times[-\pi, \pi]$, so that $D B$ is compact on $H^{0}$. In this way, the following is obtained.

THEOREM 5.3. If $v^{\prime}(s)$ is continuous and $C_{\Gamma_{c}} \frac{1}{T} 1$, then $K: H^{0} \rightarrow$ $H^{1}$ is 1-1 and onto.

The result can be extended to the equation $\left(1^{*}\right)$. By adopting $A^{-1}=-H D+J$ in equation $\left(18^{*}\right)$ the operator $D F$ then is compact on $H^{0}$ once $v^{\prime}(s)$ is continuous and $\frac{\partial}{\partial s^{\prime}} F(v(s), v(\sigma))$ is continuous on $[-\pi, \pi] \times[-\pi, \pi]$. Thus the result i) in Theorem 4.4 holds. Since for $\omega \in H_{e}^{0} \quad A \omega=A_{e} \omega$, so that $A_{e}^{-1}=A^{-1}=-H D+J$, a similar discussion will show that the result ii) in Theorem 4.4 holds. The details are omitted here.

Acknowledgements. This work was completed while the authors were visiting the University of Stuttgart. During this time I.H. Sloan 
was supported as Gastprofessor by the Deutsche Forschungsgemeinschaft. The authors are indebted to these institutions for their hospitality. They are also indebted to Dr. G.A. Chandler of the University of Queensland for suggesting the Mellin transform approach in $\S 5$ and to the Australian Research Council for its continued support.

\section{REFERENCES}

1. R.A. Adams, Sobolev Spaces, Academic Press, 1975.

2. D.N. Arnold and W.L. Wendland, On the asymptotic convergence of collocation methods, Math. Comp., Vol. 41 (1984), 349-381.

3. C.T.H. Baker, The Numerical Treatment of Integral Equations, Oxford University Press, 1977.

4. C.A. Brebbia, New Developments in Boundary Element Methods, Butterworths, 1980.

5. - Boundary Element Techniques in Computer-Aided Engineering, NATO ASI Series, 1984.

6. — Topics in Boundary Element Research, Vol. 1, Springer-Verlag, 1984.

7. - J.C.F. Telles and L.C. Wrobel, Boundary Element Techniques, Springer-Verlag, 1984.

8. G.A. Chandler, Galerkin's method for boundary integral equations on polygonal domains, J. Austral. Math. Soc. Ser. B., Vol. 26 (1984), 1-13.

9. - Numerical analysis of the boundary integral method, in Mathematical Programming and Numerical Analysis Workshop (Ed: S.A. Gustafson and R.S. Womersley), Proceedings of the Centre for Mathematical Analysis, Australian National University, Vol. 6.

10. M. Costabel, Boundary integral operators on curved polygons, Annali di Mat. pura ed appl. Ser. IVa, (1983), 305-326.

11. - E.P. Stephan and W.L. Wendland, On boundary integral equations of the first kind for the bilaplacian in a polygonal plane domain. Ann. Scuola Norm. Sup. Pisa Ser. IV, 10 (1983), 197-241.

12. and - Boundary integral equations for mixed boundary value problems in polygonal domains and Galerkin approximation. Banach Center Publications, Warsaw, 15 (1985), 175-251. Short report in: Advances in Computer Methods for Partial Differential Equations IV, IMACS (1981), 300-340.

13. and - The method of Mellin transformation for boundary integral equations on curves with corners. Numerical Solution of Singular Integral Equations. IMACS: 106 (1984), 367-413.

14. , A direct boundary integral equation method for transmission problems. J.Math. Anal. Appl. 106 (1985), 367-413.

15. - - and - On the convergence of collocation methods for boundary integral equations on polygons. Math. Comp. 49 (1987), 461-478.

16. - Boundary integral operators on Lipschitz domains. preprint No. 898 
(1985), Technische Hochschule Darmstadt, to appear in SIAM J. Math. Anal.

17. J.L. Doob, Classical Potential Theory and Its Probabilistic Counterpart, Springer-Verlag, New York, 1984.

18. G. Fairweather and R.L. Johnston, The method of fundamental solutions for problems in potential theory, in Treatment of Integral Equations by Numerical Methods (Ed. by C.T.H. Baker and G.F. Miller), Academic Press Inc. (London) Ltd.

19. D. Gaier, Integralgleichungen erster Art und konforme Abbildung, Math. Z. 147 (1976), 113-129.

20. P. Grisvard, Behaviour of the solution of an elliptic boundary value problem in a polygonal or polyhedral domain, in Numerical Solution of Partial Differential Equations III, Synspade 1975.

21. — Elliptic Problems in Nonsmooth Domains, Pitman Advanced Publishing Program, 1985.

22. G.H. Hardy and J.E. Littlewood, Some new properties of Fourier constants, in Collected Papers of G.H. Hardy III, Oxford University Press (1969), 348-399.

23. E. Hille, Analytic Function Theory, V.II., Ginn and Company, Boston, 1962.

24. G.C. Hsiao and R.C. MacCamy, Solution of boundary value problems by integral equations of the first kind, SIAM Rev. 15 (1973), 687-705.

25. — and W.L. Wendland, A finite element method for some integral equations of the first kind, J. Math. Anal. Appl. 58 (1977), 449-481.

26. - P. Kopp and W.L. Wendland, The synthesis of the collocation and Galerkin method applied to some integral equations of the first kind, in New Developments in Boundary Element Methods, C.A. Brebbia (Ed.), 1980.

27. - - - A Galerkin collocation method for some integral equations of the first kind, Computing 25 (1980), 89-130.

28. and - Some applications of a Galerkin collocation method for integral equations of the first kind, Math. Methods Appl. Sci. 6 (1984), 280-325.

29. M.A. Jaswon and G.T. Symm, Integral Equation Methods in Potential Theory and Elastostatics, Academic Press, London 1977.

30. V.A. Kondratev, Boundary problems for elliptic equations in domains with conical or angular points, Trans. Moscow Math. Soc. 16 (1967), 227-313.

31. N.S. Landkof, Foundations of Modern Potential Theory, Springer-Verlag, 1972 .

32. J.L Lions and E.Magenes, Non-homogeneous Boundary Value Problems I, Springer-Verlag, Berlin, Heidelberg, New York, 1972.

33. W. McLean, Boundary Integral Methods for the Laplace Equations, Ph.D.thesis, Australian National University, Canberra 1985.

34. S.G. Miklin, Mathematical Physics, An Advanced Course, North-Holland, Amsterdam 1970.

35. B.E. Petersen, Introduction to the Fourier Transform and Pseudo-differential Operators, Pitman Advanced Publishing Program, Boston-London-Melbourne, 1983. 
36. S. Prössdorf, Some Classes of Singular Equations, North-Holland Publishing Company, 1978.

37. J.B. Reade, Asymptotic behaviour of eigenvalues of certain integral equations, Proc. Edin. Math. Soc. 22(1979), 137-144.

38. J. Saranen and W.L. Wendland, On the asymptotic convergence of collocation methods with spline function of even degree, Math. Comp. Vol. 45 (1985), 91-108.

39. R. Seeley, Topics in pseudo-differential operators, in Pseudo-Differential Operators (L. Nirenberg, ed.), CIME, Cremonese, Roma (1969), 169-305.

40. I.H. Sloan and V. Thomee, Superconvergence of the Galerkin iterates for integral equations of the second kind, J. Integral Equations Vol. 9 (1985), 1-23.

41. - and A. Spence, The Galerkin method for integral equations of the first kind with logarithmic kernel, I. Theory, II. Application, IMA J. Numer. Anal., Vol. 8 (1988), 105-122, 123-140.

42. I.N. Sneddon, The Use of Integral Transforms, McGraw-Hill Book Company, 1972.

43. G. Verchota, Layer potentials and boundary value problems for Laplace's equations on Lipschitz domains, J. Fun. Anal. 59 (1984), 572-611.

44. W.L Wendland, Asymptotic accuracy and convergence, in Progress in Boundary Element Methods, Vol. 1, C.A. Brebbia (Ed.), Pentech Press, London, Plymouth (1981), 289-313.

45. - Boundary element methods and their asymptotic convergence. In Theoretical Acoustics and Numerical Treatments, P. Filippi (Ed.), CISM Courses and Lectures No. 277, Springer-Verlag, Wien, New York (1983), 135-216.

46. - Asymptotic accuracy and convergence for point collocation methods, in Topics in Boundary Element Research Vol. 2, C.A. Brebbia (Ed.), SpringerVerlag 1984.

47. Y. Yan and I.H. Sloan, Mesh grading for integral equations of first kind with logarithmic kernel, SIAM J. Numer. Anal., 26, No. 3 (1989).

48. A Zygmund, Trigonometric Series II, University Press, Cambridge, 1959.

School of Mathematics, University of New South Wales. Sydney. N.S.W. 2033, Australia. 
